\title{
Bevacizumab in brain tumors: ready for primetime?
}

\section{"To date, despite many efforts to establish correlation between \\ biological parameters and disease response to bevacizumab, no definitive predictor of outcome in glioblastoma patients has been demonstrated."}

\section{Enrico Franceschi†, Alicia Tosoni, Fabio Girardi \& Alba A Brandes}

${ }^{\dagger}$ Author for correspondence: Department of Medical Oncology, Azienda USL Bellaria-Maggiore, Bologna |taly = Tel.: +390516225102 = Fax: +390516225057 = e.franceschi@ausl.bo.it

Recurrent glioblastoma represents a significant issue in neuro-oncology since it remains an invariably fatal disease characterized by rapid worsening of neurological, psychological and clinical conditions of patients. Moreover, although the first-line treatment for newly diagnosed glioblastoma patients has been established by the randomized Phase III European Organisation for Research and Treatment of Cancer (EORTC) 22981/26981 - National Cancer Institute of Canada (NCIC) CE.3 trial, consisting of temozolomide concurrent with and adjuvant to radiotherapy, only a few consistent options remain for patients at the time of disease recurrence [1]. Repeat resection is not feasible and may incur unacceptable rates of morbidity in this setting, in return for a limited survival advantage, and disease always recurs within a few months. Re-irradiation should be considered for patients with relapse outside the radiotherapy field, which have been increased after the introduction of the EORTC/ NCIC treatment [2], or for patients with small lesions. However, the risk of radionecrosis remains noteworthy. Nitrosoureas have been the cornerstone of brain tumor treatment for at least 20 years, and showed a progression-free survival at 6 months (PFS-6) in the modern era of $17.5 \%$ [3]. Moreover, only a few activity data of these compounds (i.e., fotemustine) after radiotherapy and temozolomide treatment are available, showing a PFS- 6 of $21 \%$ [4].

\footnotetext{
"Recurrent glioblastoma represents

a significant issue in neuro-oncology since it remains an invariably fatal disease..."
}

In recent years, novel approaches have been developed, and antiangiogenic treatments now have a relevant role in the treatment of colorectal, renal, breast and lung cancers.
Malignant gliomas produce multiple angiogenic factors to induce neovascularization by angiogenesis, vasculogenesis and vascular remodeling. Moreover, high-grade gliomas are vascular tumors and preclinical data indicate that angiogenesis is essential for proliferation and survival of malignant glioma cells, and the VEGF pathway appears to play a particularly important role. Therefore, after a first experience in 2005 [5], which showed an impressive response rate of $66 \%$ and a limited toxicity in 29 recurrent malignant gliomas treated with a VEGF-neutralizing antibody, bevacizumab $\left(\right.$ Avastin $\left.^{\circledR}\right)$, administered in combination with irinotecan, some other groups published retrospective data and prospective studies as single agent or in combination, with response rates that ranged from $20-60 \%$, and PFS- 6 rates that ranged from $25-50 \%$ [6-10]. These data represented something never before observed in neuro-oncology, and raised the interest of the scientific community. Based on the durable objective response rates observed in the AVF3708g and NCI 06-C-0064E trials [7,10], which evaluated the role of bevacizumab alone or in combination with irinotecan, the US FDA granted accelerated approval to bevacizumab as a single agent for patients with glioblastoma. Some points remain to be clarified. Since VEGF regulates vascular permeability, targeting VEGF with bevacizumab may reduce contrast leakage into the tumor, thus producing neuroradiological pictures of maximized radiographic response, or also pseudoresponses with the decreased contrast enhancement that makes complex the real disease response assessment, and should need further improvement in neuroradiology as well as the implementation with other imaging techniques [11].

To date, despite many efforts to establish correlation between biological parameters and disease response to bevacizumab, no definitive predictor of outcome in glioblastoma patients has been demonstrated. Nevertheless, overall 
survival data from Phase II studies, ranging from 7-10 months even in heavily pretreated recurrent glioblastoma patients, are intriguing and have warranted the prosecution of clinical research with other Phase II and novel Phase III trials, to evaluate the best companion drug for bevacizumab and to evaluate the impact on overall survival of this antiangiogenic compound. Moreover, due to the results obtained in the recurrence setting, bevacizumab is also under investigation for the treatment of newly diagnosed glioblastoma patients.

\begin{tabular}{c}
\hline "Based on the durable objective \\
response rates observed in the AVF3708g \\
and NCl 06-C-0064E trials ... the US FDA \\
granted accelerated approval to \\
bevacizumab as a single agent for \\
patients with glioblastoma."
\end{tabular}

The forthcoming new BO21990 Phase III trial for newly diagnosed glioblastoma in which bevacizumab will be added to standard therapy in the experimental BO21990 arm will drive a definitive conclusion regarding the therapeutic role of bevacizumab and if we really have a new standard therapy for this dismal tumor.

Finally, the introduction of bevacizumab and other antiangiogenic compounds (i.e., cediranib and aflibercept) has raised interest in further research in a field (i.e., the recurrence glioblastoma setting) where noteworthy results were not obtained by cytotoxic approaches, providing encouraging results and new hopes for brain tumor patients.

\section{Financial \& competing interests disclosure}

The authors have no relevant affiliations or financial involvement with any organization or entity with a financial interest in or financial conflict with the subject matter or materials discussed in the manuscript. This includes employment, consultancies, honoraria, stock ownership or options, expert testimony, grants or patents received or pending, or royalties.

No writing assistance was utilized in the production of this manuscript.

\section{Bibliography}

Papers of special note have been highlighted as:

- of interest

." of considerable interest

1. Stupp R, Mason WP, van den Bent MJ et al.: Radiotherapy plus concomitant and adjuvant temozolomide for glioblastoma. N. Engl. J. Med. 352(10), 987-996 (2005).

- Milestone paper that changed the state-of-the-art treatment for glioblastoma.

2. Brandes AA, Tosoni A, Franceschi E et al.: Recurrence pattern after temozolomide concomitant with and adjuvant to radiotherapy in newly diagnosed glioblastoma patients: correlation with MGMT promoter methylation status. J. Clin. Oncol. 27(8), 1275-1279 (2009).

3. Brandes AA, Tosoni A, Amista P et al: : How effective is BCNU in recurrent glioblastoma in the modern era? A Phase II trial. Neurology 63(7), 1281-1284 (2004).

4. Brandes AA, Tosoni A, Franceschi E et al.: Fotemustine as second-line treatment for recurrent or progressive glioblastoma after concomitant and/or adjuvant temozolomide: a Phase II trial of Gruppo Italiano Cooperativo di Neuro-Oncologia (GICNO). Cancer Chemother. Pharmacol. 64(4), 769-775 (2009).

5. Stark-Vance V: Bevacizumab and CPT-11 in the treatment of relapsed malignant glioma.
In: World Federation of Neuro-Oncology Second Quadrennial Meeting. Edinburgh, UK, May 5-8 (2005) (Abstract 342).

6. Bokstein F, Shpigel S, Blumenthal DT: Treatment with bevacizumab and irinotecan for recurrent high-grade glial tumors. Cancer 112(10), 2267-2273 (2008).

7. Cloughesy TF, Prados MD, Wen PY et al.: A Phase II, randomized, non-comparative clinical trial of the effect of bevacizumab (BV) alone or in combination with irinotecan (CPT) on 6-month progressionfree survival (PFS6) in recurrent, treatmentrefractory glioblastoma (GBM). J. Clin. Oncol. (Meeting Abstracts), 26(15 Suppl.) (2008) (Abstract 2010b).

- One of the trials evaluated by the US FDA to grant accelerated approval to bevacizumab.

8. Vredenburgh JJ, Desjardins A, Herndon JE 2nd et al:: Bevacizumab plus irinotecan in recurrent glioblastoma multiforme. J. Clin. Oncol. 25(30), 4722-4729 (2007).

9. Vredenburgh JJ, Desjardins A, Herndon JE, 2nd et al:: Phase II trial of bevacizumab and irinotecan in recurrent malignant glioma. Clin. Cancer. Res. 13(4), 1253-1259 (2007).

10. Kreisl TN, Kim L, Moore K et al.: Phase II trial of single-agent bevacizumab followed by bevacizumab plus irinotecan at tumor progression in recurrent glioblastoma. J. Clin. Oncol. 27(5), 740-745 (2008).
- One of the trials evaluated by the US FDA to grant accelerated approval to bevacizumab.

11. Chen W, Delaloye S, Silverman DH et al: Predicting treatment response of malignant gliomas to bevacizumab and irinotecan by imaging proliferation with $[18 \mathrm{~F}]$ fluorothymidine positron emission tomography: a pilot study. J. Clin. Oncol. 25(30), 4714-4721 (2007).

\section{Affiliations}

- Enrico Franceschi

Medical Oncology Department, Bellaria-Maggiore Hospital, Bologna, Italy Tel.: +39051622 5102 Fax: +390516225057 e.franceschi@ausl.bo.it

- Alicia Tosoni Medical Oncology Department, Bellaria-Maggiore Hospital, Bologna, Italy

- Fabio Girardi Medical Oncology Department, Bellaria-Maggiore Hospital, Bologna, Italy

- Alba A Brandes Medical Oncology Department, Bellaria-Maggiore Hospital, Bologna, Italy 